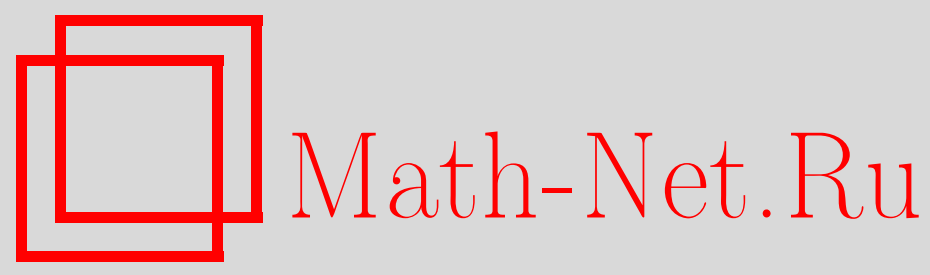

K. А. Шрамов, Элементарные бирациональные отображения между трехмерными торическими расслоениями Мори, Матем. заметки, 2005, том 78, выпуск 1, 132-139

DOI: https://doi.org/10.4213/mzm2567

Использование Общероссийского математического портала Math-Net.Ru подразумевает, что вы прочитали и согласны с пользовательским соглашением http://www.mathnet.ru/rus/agreement

Параметры загрузки:

IP : 54.209 .52 .79

26 апреля 2023 г., 14:46:55

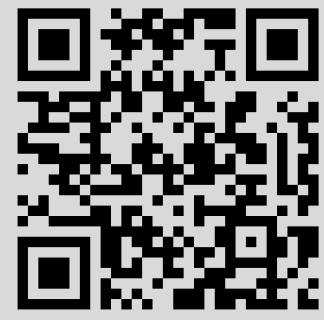


том 78 выпуск 1 июль 2005

УДК 512.763

\section{ЭЛЕМЕНТАРНЫЕ БИРАЦИОНАЛЬНЫЕ ОТОБРАЖЕНИЯ МЕЖДУ ТРЕХМЕРНЫМИ ТОРИЧЕСКИМИ \\ РАССЛОЕНИЯМИ МОРИ}

\section{К. А. Шрамов}

В данной работе классифицируются элементарные эквивариантные бирациональные отображения - линки - между трехмерными торическими Мори расслоениями. Все они естественно подразделяются на несколько классов в зависимости от размерностей баз расслоений. В тех случаях, когда количество линков оказьвается конечньм, приводится их полный список, в других случаях дается их локальное описание (см. формулировки в разделе 4). Почти все доказательства комбинаторные, поэтому доказьваются лишь вспомогательные утверждения раздела 3 .

Библиограбфия: 5 названий.

1. Введение. Напомним (см., например, [1]), что Мори расслоенное пространство (Мори расслоение) - это многообразие $X$ с отображением $\phi: X \rightarrow S$, обладающее следующими свойствами:

(i) $X$ проективно, $\mathbb{Q}$-факториально и имеет не более чем терминальные особенности;

(ii) $\phi: X \rightarrow S$ - стягивание $K_{X}$-отрицательного экстремального луча;

(iii) $\operatorname{dim}(S)<\operatorname{dim}(X)$.

Ответ на вопрос о том, как устроены бирациональные отображения между Мори расслоенными пространствами, дает алгоритм разложения таких отображений на элементарные (назьваемые линками), известный под названием программы Саркисова (см., например, [1]).

Линками типа I, II, III $u$ IV называются коммутативные диаграммы следующего вида:
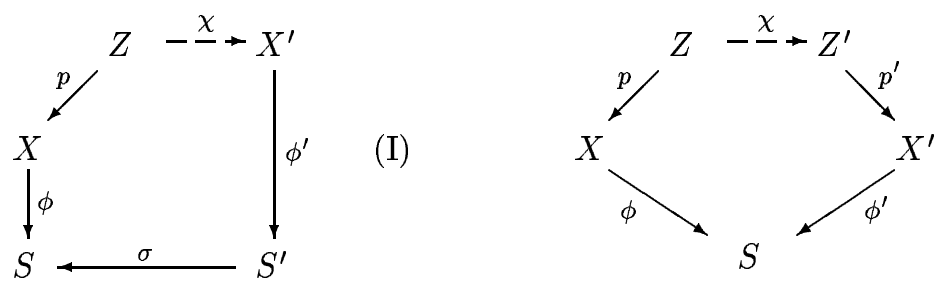

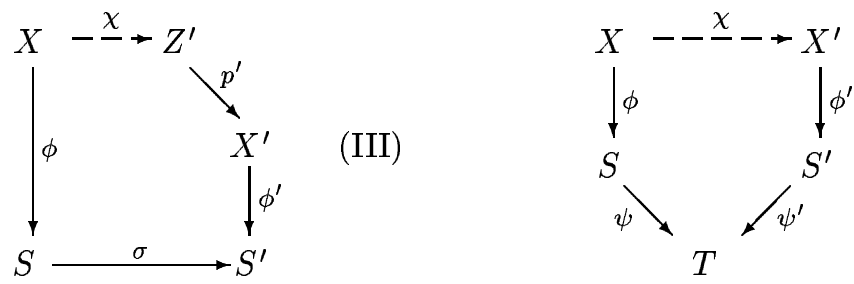

В каждой из этих диаграмм отображения $\phi: X \rightarrow S$ и $\phi^{\prime}: X^{\prime} \rightarrow S^{\prime}-$ Мори расслоения, отображения $p: Z \rightarrow X$ и $p^{\prime}: Z^{\prime} \rightarrow X^{\prime}$ - экстремальные дивизориальные стягивания, отображение $\chi$ является последовательностью лог-флипов (быть может, тривиальной), $\sigma, \psi$ и $\psi^{\prime}-$ сюръективные морфизмы со связньми слоями. Особенности многообразий $X, X^{\prime}, Z$ и $Z^{\prime}$ всегда предполагаются терминальными.

Доказательства основных фактов, относяшихся к программе Саркисова в размерностях 2 и 3, можно найти в [1].

2. Обозначения и соглашения. В дальнейшем все многообразия предполагаются проективными, нормальньги и $\mathbb{Q}$-факториальными. Основное поле - поле комплексных чисел $\mathbb{C}$.

Определения и информация об основных свойствах торических многообразий содержатся, например, в [1] или [2].

Для каждого $n$-мерного торического многообразия $M$ символ $\mathbb{F}_{M} \subset \mathcal{N} \otimes_{\mathbb{Z}} \mathbb{Q}, \mathscr{N}=\mathbb{Z}^{n}$, обозначает его веер, а $\mathbb{F}_{M}^{(k)}$ - множество $k$-мерных конусов этого веера. Все вееры считаются вложенньпи в одно и то же пространство, чтобы некоторые их элементы можно было отождествлять друг с другом. Элементы $\mathbb{F}_{M}^{(1)}$, т.е. одномерные конусы, для сокрашения формулировок отождествляются с лежащими на них примитивными векторами. Конус из $\mathbb{F}_{M}^{(k)}$, порожденньй векторами $v_{1}, \ldots, v_{k}$, обозначается $\left\langle v_{1}, \ldots, v_{k}\right\rangle$.

При рассмотрении линков предполагается, что обозначения всех многообразий и отображений такие же, как в определениях (см. выше). Все линки рассматриваются с точностью до послойного изоморфизма.

Основное предположение: если противное не оговорено явно, то все объекты предполагаются торическими.

3. Специфика торической программы Саркисова. Обсуждение торической программы Саркисова можно найти в [1]. Отметим лишш то, что любые два торических многообразия одинаковой размерности бирационально эквивалентны, так что алгоритм можно применять к любой паре торических Мори расслоений.

Торические многообразия обладают рядом свойств, сильно упрощающих описание элементов программы Саркисова. Ниже перечислены некоторые из них.

Лемма 1. Пусть $X$ - гладкое трехмерное торическое многообразие, $\phi: X \rightarrow$ $S=\mathbb{P}^{1}$ - әквивариантное расслоение со связными слоями и $\rho(X / S)=1$. Тогда $X=\mathbb{P}_{\mathbb{P}^{1}}(\mathscr{O} \oplus \mathscr{O}(a) \oplus \mathscr{O}(b))$ для некоторьх $a, b \in \mathbb{Z}$.

ДоказАтЕльство. Так как $\rho(X / S)=1$, то $\rho(X)=2$. Пусть $\mathbb{F}_{X}^{(1)}=\left\{v_{1}, \ldots, v_{5}\right\}$, причем векторы $v_{1}, v_{2}$ и $v_{3}$ лежат в плоскости $P$, проекция вдоль которой переводит $\mathbb{F}_{X}$ в $\mathbb{F}_{S}$. Так как точки, соответствующие $\left\langle v_{1}, v_{2}, v_{4}\right\rangle$ и $\left\langle v_{1}, v_{2}, v_{5}\right\rangle$, гладкие, можно считать $v_{1}=(1,0,0), v_{2}=(0,1,0), v_{3}=(-1,-1,0), v_{4}=(0,0,-1), v_{5}=(a, b, 1)$. 
ЗАмЕчАнИЕ 1. Предыдущее утверждение остается верным и при более слабых условиях на $X$ : достаточно требовать терминальности особенностей и существования двух гладких инвариантных точек, лежащих на различных слоях. В частности, из этих условий следует гладкость $X$. Именно в таком виде это утверждение удобно использовать в дальнейшем.

Терминальность особенностей накладывает сильные ограничения на торические Мори расслоения. Именно, имеют место следуюшие утверждения, которые используются для локального описания линков над базами, отличными от точки.

Лемма 2. Пусть $\tilde{X}=\mathbb{P}^{2} \times \mathbb{A}^{1} / \mathbb{Z}_{n}(a: b: 0,1)$, дде $n>1,(a, n)=(b, n)=1$, uмеет лишь терминальные особенности. Тогда $n=3, a=2, b=1$ (или $a=1, b=2)$.

ДокАЗАтЕльство. Многообразие $\widetilde{X}$ имеет три особые точки типа $\frac{1}{n}(a, b, 1), \frac{1}{n}(a-b$, $-b, 1)$ и $\frac{1}{n}(-a, b-a, 1)$ соответственно. Так как первая из них терминальна, то возможны лишь следующие случаи.

Случай $b=-a$. Тогда, чтобы особая точка типа $\frac{1}{n}(a-b,-b, 1)=\frac{1}{n}(2 a, a, 1)$ была терминальна, необходимо либо $a=-1$ (тогда из терминальности точки типа $\frac{1}{n}(-a, b-a$, $1)=\frac{1}{n}(1,2,1)$ следует, что $n=3$ ), либо $2 a=-1$ (тогда из терминальности точки типа $\frac{1}{n}(-a, b-a, 1)=\frac{1}{n}(-a, 1,1)$ следует $a=1$, т.е. $\left.n=3\right)$, либо $a=-2 a$ (тогда $3 a=0$, и из $(a, n)=1$ следует $n=3)$.

Случай $a=-1$. Тогда, чтобы особая точка типа $\frac{1}{n}(a-b,-b, 1)=\frac{1}{n}(-1-b,-b, 1)$ была терминальна, необходимо либо $-b=-1$ (тогда $b=1$, и из терминальности точки типа $\frac{1}{n}(-a, b-a, 1)=\frac{1}{n}(1,2,1)$ следует $\left.n=3\right)$, либо $-1-b=-1$ (тогда $b=0$; противоречие), либо $-1-b=b$ (тогда $2 b=-1$, что дает $b=1$, и $n=3$ ).

Случай $b=-1$ разбирается аналогично.

Лемма 3. Пусть $\phi: X \rightarrow S=\mathbb{P}^{1}$ - трехмерное торическое Мори расслоение, $\widetilde{S}=\mathbb{A}^{1} \subset S$ и $\widetilde{X}=\phi^{-1}(\widetilde{S})$. Тогда $\widetilde{X}$ изоморфно либо $\mathbb{P}^{2} \times \mathbb{A}^{1}$, либо $\mathbb{P}^{2} \times \mathbb{A}^{1} / \mathbb{Z}_{3}(2$ : $1: 0,1)$.

ДокАЗАтЕльство. То, что $\widetilde{X}$ является фактором $\mathbb{P}^{2} \times \mathbb{A}^{1}$ (или совпадает с ним), следует из строения веера торического многообразия $X$. То, что соответствующая группа циклическая, следует из терминальности особенностей $X$. Оставшаяся часть утверждения содержится в лемме 2 .

Лемма 4. Пусть $\widetilde{X}=\mathbb{P}^{1} \times \mathbb{P}^{1} \times \mathbb{A}^{1} / \mathbb{Z}_{n}(a: 0, b: 0,1)$, дде $n>1,(a, n)=(b, n)=1$, имеет лишь терминальные особенности. Тогда $n=2, a=b=1$.

ДокАЗАтЕльство. Многообразие $\widetilde{X}$ имеет особые точки типа $\frac{1}{n}(a, b, 1), \frac{1}{n}(a,-b, 1)$, $\frac{1}{n}(-a, b, 1)$ и $\frac{1}{n}(-a,-b, 1)$ соответственно. Разбор случаев устроен так же, как в доказательстве леммы 2 .

ЗАмЕчАниЕ 2. Аналогичное лемме 4 локальное по базе описание трехмерных торических расслоений на коники таково: проекция $\phi: \widetilde{X} \rightarrow \widetilde{S}$ есть либо $\mathbb{P}^{1} \times \mathbb{A}^{2} \rightarrow \mathbb{A}^{2}$, либо $\mathbb{P}^{1} \times \mathbb{A}^{2} / \mathbb{Z}_{n}(a,-a, 1) \rightarrow \mathbb{A}^{2} / \mathbb{Z}_{n}(1,-1)$, где $n>1,(a, n)=1$.

Для описания дивизориальных стягиваний в линках используется следующая теорема, доказанная в [3].

Теорема (Кавамата [3]). Пусть $(X, P)$ - факторособенность $\mathbb{C}^{3} / \mathbb{Z}_{r}(s,-s, 1), 0<$ $s<r,(r, s)=1, u \mu: Y \rightarrow X-$ проективный бирациональный морфизм из многообразия $Y$ с терминальными особенностями. Предположсим, что исключительное множество $Е$ морфизма $\mu$ является неприводимым $\mathbb{Q}-$ Картье дивизором, $-E$ 
$\mu$-обилен и $P \in \mu(E)$. Тогда $\mu$ совпадает со взвешенным раздутием $P$ с весами $(s / r,(r-s) / r, 1 / r)$.

Имеется полньй список трехмерных торических Мори расслоений над точкой (т.е. многообразий $\mathbb{Q}$ - $а$ ано с группй Пикара $\mathbb{Z}$ ) с терминальньми особенностями (см. [4]). Это следующие многообразия: $\mathbb{P}^{3}, \mathbb{P}(1,1,1,2), \mathbb{P}(1,1,2,3), \mathbb{P}(1,2,3,5), \mathbb{P}(1,3,4,5), \mathbb{P}(2$, $3,5,7), \mathbb{P}(3,4,5,7)$, а также торическое многообразие $\mathscr{V}$, для которого примитивные векторы одномерных конусов веера $\mathbb{F}_{\mathscr{V}}$ суть $(1,0,1),(-2,1,1),(1,-2,0)$ и $(0,1,-2)$ (это многообразие является фактором $\mathbb{P}^{3}$ по групе $\left.\mathbb{Z}_{5}\right)$.

4. Формулировки утверждений о торических линках. В следующемпредложении описьваются торические линки типа I. Так как линки типа III являются обратными преобразованиями к линкам типа I, их описание отличается от описания линков типа I лишь переобозначениями.

ПРЕДЛОЖЕНИЕ 1. Пусть $X \rightarrow S, X^{\prime} \rightarrow S^{\prime}-$ трехмерные торические Мори расслоения. Тогда для линков типа I имеются лишь следующие возможности.

1) $\rho(X)=1, S=p t, \operatorname{dim}\left(S^{\prime}\right)=2, p-$ раздутие точки, $\chi-$ изоморфизм. Все торические линки такого вида исчерпываются приведенным ниже списком из шести примеров.

а) Раздутие точки на $X=\mathbb{P}^{3}$.

б) Пусть $X=\mathbb{P}(1,1,1,2)$. Раздутие гладкой точки на $X$ приводит к расслоению на коники над $\mathbb{P}(1,1,2)$. Раздутие особой точки (особенность типа $\left.\frac{1}{2}(1,1,1)\right)$ приводит $к$ неособому расслоению над $\mathbb{P}^{2}$.

в) Пусть $X=\mathbb{P}(1,1,2,3)$. Взвешенное раздутие особой точки типа $\frac{1}{3}(1,2,1)$ c весами $\left(\frac{1}{3}, \frac{2}{3}, \frac{1}{3}\right)$ приводит к расслоению над $\mathbb{P}(1,1,2)$. Взвешенное раздутие гладкой точки с весами $(1,2,3)$ приводит $к$ расслоению над $\mathbb{P}(1,2,3)$.

г) Пусть $X=\mathbb{P}(1,2,3,5)$. Взвешенное раздутие особой точки типа $\frac{1}{5}(1,2,3)$ приводит к расслоению над $\mathbb{P}(1,2,3)$.

2) $\rho(X)=1, S=p t, \operatorname{dim}\left(S^{\prime}\right)=1, p$ - раздутие точки, $\chi$ - один лог-флип. Все торические линки такого вида исчерпываются приведенным ниже списком из четырех примеров.

а) Пусть $X=\mathbb{P}(1,1,1,2)$. Раздутие гладкой точки $P \in X$ приводит к многообразию $Z$ с одной терминальной особенностью. При этом $\rho(Z)=2$, один из әкстремальных лучей конуса $N E(Z)$ порожден кривой на исключительном дивизоре стягивания $p$. Пусть $D_{1}, D_{2} \subset X$ - неприводимые инвариантные дивизоры, содержсащие $P$ и особую точку $P^{\prime} \in X$. Пусть $\widetilde{D}_{i}=p_{*}^{-1} D_{i}, i=1,2, C=\widetilde{D}_{1} \cap \widetilde{D}_{2}$. Тогда C порождает әкстремальный луч, $K_{Z} C=-3 / 2$, и флип в $C$ приводит $\kappa$ неособому многообразию $X^{\prime}=\mathbb{P}_{\mathbb{P}^{1}}(\mathscr{O} \oplus \mathscr{O}(1) \oplus \mathscr{O}(2))$.

б) Пусть $X=\mathbb{P}(1,1,2,3)$. Раздутие особой точки $P_{2}$ типа $\frac{1}{2}(1,1,1)$ с последуюшим лог-флипом в пересечении собственных прообразов неприводимых инвариантных дивизоров, содерэсащих $P_{2}$ и особую точку $P_{3}$ индекса 3 , приводит к многообразию $X=\mathbb{P}_{\mathbb{P}^{1}}(\mathscr{O} \oplus \mathscr{O}(1) \oplus \mathscr{O}(3))$. Взвешенное раздутие гладкой точки с весами $(1,1,3)$ приводит к особому Мори расслоению.

в) Пусть $X=\mathbb{P}(1,3,4,5)$. Взвешенное раздутие особой точки индекса 4 приводит к особому Мори расслоению.

3) $\rho(X)=1, S=p t, \operatorname{dim}\left(S^{\prime}\right)=1, p-$ раздутие кривой, $\chi-$ изоморфизм. Единственный пример такого линка - раздутие прямой $l \subset \mathbb{P}^{3}$. 
4) $\rho(X)=2, \operatorname{dim}(S)=1, \operatorname{dim}\left(S^{\prime}\right)=2, p-$ раздутие сюрвективно проецирующейся на $S$ кривой, $\chi$ - изоморфизм. Таких линков бесконечно много, все участвующие в них многообразия гладкие, и все они могут быть описаны следующим образом.

Пусть $X=\mathbb{P}_{\mathbb{P}_{1}}(\mathscr{O} \oplus \mathscr{O}(a) \oplus \mathscr{O}(b)), a, b \in \mathbb{Z}, a \geqslant b$. Пусть $M-$ тавтологический обратимый пучок на $X$, дивизор $L-$ слой расслоения $\phi: X \rightarrow \mathbb{P}^{1}$. Пусть кривая $s$ - сечение, соответствующее проекиии расслоения $\mathscr{O} \oplus \mathscr{O}(a) \oplus \mathscr{O}(b)$ на $\mathscr{O}$. Пусть $p: X^{\prime} \rightarrow X-$ раздутие кривой $s$. Тогда $S^{\prime}=F_{a-b}=\mathbb{P}_{\mathbb{P}^{1}}(\mathscr{O} \oplus \mathscr{O}(a-b))$, а отображение $\sigma: S^{\prime} \rightarrow S$ - соответствующее $\mathbb{P}^{1}$-расслоение $F_{a-b} \rightarrow \mathbb{P}^{1}$. Исключительный дивизор Е отображсения р проецируется на $S^{\prime}$ изоморфно.

Представляя $X$ в таком виде различными способами (число способов не более трех), получаем все возможсности для $X^{\prime}$.

5) $\rho(X) \geqslant 2, \operatorname{dim}(S)=2, \operatorname{dim}\left(S^{\prime}\right)=2, p-$ раздутие точки, $\chi$ - один лог-флип. Таких линков бесконечно много, и им мохно дать следующее локальное (по $S$ ) описание. Если $X / S$ локально изоморфно $\mathbb{A}^{2} \times \mathbb{P}^{1} / \mathbb{A}^{2}$, то $p$-взвешенное раздутие $c$ весами $(a, 1, b),(a, b)=1$, соответствующее отображсение баз $\sigma$ - взвешенное раздутие с весами $(a, 1)$. Если же $X / S$ локально изоморфно $\left(\mathbb{A}^{2} \times \mathbb{P}^{1} / \mathbb{Z}_{r}(s,-s, 1: 0)\right) /$ $\left(\mathbb{A}^{2} / \mathbb{Z}_{r}(1,-1)\right)$, то единственная возможнность для $р$ описывается теоремой $K a-$ ваматы (см. раздел 3), соответствующее отображение баз - взвешенное раздутие с весами $(s / r,(r-s) / r, 1 / r)$.

6) $\rho(X) \geqslant 2, \operatorname{dim}(S)=2, \operatorname{dim}\left(S^{\prime}\right)=2, p-$ раздутие слоя ф над гладкой точкой, $\chi$ - изоморфизм, отображсение $\sigma: S^{\prime} \rightarrow S$ - раздутие соответствующей точки на базе.

СХемА дОкАЗАТЕЛЬСТВА. Пусть $S=p t$. Пусть $\mathbb{F}_{X}^{(1)}=\left\{v_{1}, \ldots, v_{4}\right\}, \mathbb{F}_{Z}^{(1)}=\mathbb{F}_{X}^{(1)} \cup$ $\left\{v_{5}\right\}$. Предположим, что $\operatorname{dim}\left(S^{\prime}\right)=2$. Тогда, так как существует проекция $\mathbb{F}_{X^{\prime}}$ на $\mathbb{F}_{S^{\prime}}$, веер $\mathbb{F}_{X^{\prime}}$ должен содержать прямую. Так как $\mathbb{F}_{X^{\prime}}^{(1)}=\mathbb{F}_{Z}^{(1)}$, отсюда следует, что $v_{5}=-v_{i}$ для некоторого $i$, в частности, $p$ - стягивание в точку. Окончательное описание в этом случае получается в результате небольшого перебора, выделяющего среди всех возникающих так многообразий имеющие лишь терминальные особенности.

Предположим теперь, что $\operatorname{dim}\left(S^{\prime}\right)=1$. Тогда $\mathbb{F}_{X^{\prime}}$ проецируется на $\mathbb{F}_{S^{\prime}}=\mathbb{F}_{\mathbb{P}^{1}}$ вдоль плоскости, и три вектора из $\mathbb{F}_{X^{\prime}}^{(1)}=\mathbb{F}_{Z}^{(1)}$, например, $v_{3}, v_{4}$ и $v_{5}$, должны быть компланарны. Так как $X^{\prime}$ имеет лишь терминальные особенности, то $v_{5}=-\left(v_{3}+v_{4}\right)$, что снова дает конечное количество возможностей для $Z$. При этом то, является ли $p$ стягиванием на кривую или в точку, в терминах $\mathbb{F}_{Z}$ определяется тем, компланарны $v_{5}, v_{1}$ и $v_{2}$ или нет.

Пусть теперь $\operatorname{dim}(S)=1$, т.е. $S=\mathbb{P}^{1}$. Из коммутативности диаграммы, описьвающей линк, и строения $\mathbb{F}_{X}$ нетрудно видеть, что образ исключительного дивизора $p$ не может содержаться в слое $\phi$, т.е. это с необходимостью кривая, сюръективно проецирующаяся на $S$. По теореме Каваматы она не проходит через особые точки $X$, а по лемме 1 (см. замечание 1) отсюда следует, что $X=\mathbb{P}_{\mathbb{P}^{1}}(\mathscr{O} \oplus \mathscr{O}(a) \oplus \mathscr{O}(b))$.

В оставшихся случаях $(\operatorname{dim}(S)=2)$ из строения $\mathbb{F}_{X}$ очевидно, что $p$ должно быть либо стягиванием на слой $\phi$ (тогда по теореме Каваматы такой слой не проходит через особые точки $X$ ), либо стягиванием в точку. Дальнейшее описание получается применением теоремы Каваматы к локальному описанию торических расслоений на коники.

ЗАмЕчАниЕ 3. Многие утверждения о тривиальности (нетривиальности) отображения $\chi$ в общем (неторическом) случае перестают быть верными. Например, раздутие кривой степени 7 рода 5 на $\mathbb{P}^{3}$ является расслоением на коники (в торическом случае 
раздутие кривой на многообразии $X$ с $\rho(X)=1$ может привести только к расслоению над $\left.\mathbb{P}^{1}\right)$.

Наиболее многочис ленную группу составляют линки типа II (для перечисления линков из пункта 1) предложения 2 был использован компьютерньй перебор).

ПРЕДЛОЖЕНИЕ 2. Пусть $X \rightarrow S, X^{\prime} \rightarrow S^{\prime}-$ трехмерные торические Мори расслоения. Тогда для линков типа II имеются лишь следующие возможности.

1) $\rho(X)=\rho\left(X^{\prime}\right)=1, S=p t, p, p^{\prime}$ - раздутия точек, $\chi$ - один лог-флип. Все торические линки такого вида исчерпываются приведенным ниже списком из тридиати восьми (содержательно - девятнадиати) примеров.

а) Пусть $X=\mathbb{P}^{3}$. Если $p$ - взвешенное раздутие с весами $(1,2,3)$, то описанный линк приводит $\kappa X^{\prime}=\mathbb{P}(1,1,2,3)$. Если $p$ - взвешенное раздутие $c$ весами $(1,2,5)$, то $X^{\prime}=\mathbb{P}(1,3,4,5)$.

б) Пусть $X=\mathbb{P}(1,1,1,2)$. При некотором выборе координат взвешенные раздутия гладкой точки с весами $(2,1,1),(3,1,1),(2,1,3),(4,1,3),(2,1,5)$ и $(2,1,7)$ приводят посредством описанного линка $к$ многообразиям $\mathbb{P}(1,1$, $2,3), \mathbb{P}(1,2,3,5), \mathbb{P}(1,1,1,2), \mathbb{P}(1,3,4,5), \mathbb{P}(1,2,3,5)$ и $\mathbb{P}(2,3,5,7)$ соответственно.

в) Пусть $X=\mathbb{P}(1,1,2,3)$. При некотором выборе координат взвешенные раздутия гладкой точки с весами $(1,1,1),(2,1,1),(1,1,2),(1,3,2),(1,3,4)$, $(1,5,4),(1,3,5),(1,3,7),(1,1,4) u(1,1,5)$ приводят посредством описанного линка к многообразиям $\mathbb{P}(1,1,1,2), \mathbb{P}(1,2,3,5), \mathbb{P}^{3}, \mathbb{P}(1,2,3,5), \mathbb{P}(1,1,2,3)$, $\mathbb{P}(2,3,5,7), \mathbb{P}(1,2,3,5), \mathbb{P}(3,4,5,7), \mathbb{P}(1,3,4,5)$ и $\mathbb{P}(2,3,5,7)$ соответственно.

г) Пусть $X=\mathbb{P}(1,2,3,5)$. При некотором выборе координат взвешенные раздутия гладкой точки с весами $(1,1,1),(1,1,2),(1,2,3),(1,1,3),(1,1,4) u$ $(1,2,5)$ приводят посредством описанного линка к многообразиям $\mathbb{P}(1,1$, $2,3), \mathbb{P}(1,1,1,2), \mathbb{P}(1,1,2,3), \mathbb{P}(1,3,4,5), \mathbb{P}(3,4,5,7)$ и V соответственно, а взвешенные раздутия особых точек индекса 2 и 3 - к многообразиям $\mathbb{P}(1,1,1,2) u \mathbb{P}(1,1,2,3)$.

д) Пусть $X=\mathbb{P}(1,3,4,5)$. При некотором выборе координат взвешенные раздутия гладкой точки с весами $(1,1,1),(2,1,1),(1,3,2),(1,1,2) u(1,1,3)$ приводят посредством описанного линка к многообразиям $\mathbb{P}(1,1,2,3), \mathbb{P}(2$, $3,5,7), \mathbb{P}(3,4,5,7), \mathbb{P}(1,2,3,5)$ и $\mathbb{P}(3,4,5,7)$ соответственно, а взвешенные раздутия особых точек индекса 3 и 5 - кмногообразиям $\mathbb{P}^{3} u \mathbb{P}(1,1,1,2)$.

е) Пусть $X=\mathbb{P}(2,3,5,7)$. Тогда взвешенные раздутия особых точек индекса $2,3,5$ и 7 приводят посредством описанного линка $к$ многообразиям $\mathbb{P}(1,1,2,3), \mathbb{P}(1,1,1,2), \mathbb{P}(1,3,4,5)$ и $\mathbb{P}(1,1,2,3)$ соответственно.

ж) Пусть $X=\mathbb{P}(3,4,5,7)$. Тогда взвешенные раздутия особых точек индекса $3,4,5$ и 7 приводят посредством описанного линка $к$ многообразиям $\mathbb{P}(1,2,3,5), \mathbb{P}(1,3,4,5), \mathbb{P}(1,1,2,3)$ u $\mathbb{P}(1,3,4,5)$ соответственно.

з) Пусть $X=\mathscr{V}$. Тогда взвешенное раздутие особой точки приводит посредством описанного линка $к$ многообразию $\mathbb{P}(1,2,3,5)$.

2) $\rho(X)=\rho\left(X^{\prime}\right)=1, S=p t$, одно из отображсений $p, p^{\prime}$ - раздутие кривой, другое - раздутие точки, $\chi$ - изоморфизм. Существует лишь два торических линка такого вида. Первый связывает многообразия $X=\mathbb{P}(1,1,1,2)$ и $X^{\prime}=\mathbb{P}^{3}$, причем $p^{\prime}$ - взвешенное раздутие с весами $(1,1,2)$. Второй связывает $X=\mathbb{P}(1,1,2,3) u$ $X^{\prime}=\mathbb{P}(1,1,1,2)$, причем $p^{\prime}$ - взвешенное раздутие гладкой точки с весами $(1,1,3)$. 
3) $\rho(X)=\rho\left(X^{\prime}\right)=2, \operatorname{dim}(S)=1, p, p^{\prime}-$ раздутия точек, $\chi$ - один лог-флип. Таких линков бесконечно много, и им можсно дать следующее локальное описание. Пусть $X / S$ локально (по $S$ ) изоморфно $\mathbb{P}^{2} \times \mathbb{A}^{1} / \mathbb{A}^{1}$. Тогда $p$ - либо взвешенное раздутие с весами $(a, b, 1) \neq(1,1,1),(a, b)=1$, причем в результате снова получается (локально) гладкое многообразие $X^{\prime}$, либо взвешенное раздутие с весами $(1,3 a+2,3), a \geqslant 0$, причем в результате получается особое многообразие. Ecли же $X / S$ локально изоморфно $\left(\mathbb{P}^{2} \times \mathbb{A}^{1} / \mathbb{Z}_{3}(2: 1: 0,1)\right) / \mathbb{A}^{1}$, то р описывается теоремой Каваматы и $X^{\prime}$ (локально) гладко.

4) $\rho(X)=\rho\left(X^{\prime}\right)=2, \operatorname{dim}(S)=1$, одно из отображсений $p, p^{\prime}-$ раздутие точки,

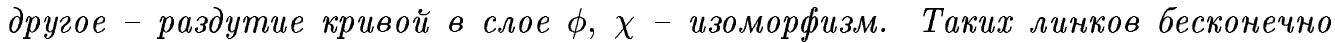
много, участвующие в них многообразия гладкие, и им мохно дать локальное $\left(\right.$ по $S$ ) описание: $X / S$ локально изоморфно $\mathbb{P}^{2} \times \mathbb{A}^{1} / \mathbb{A}^{1}$, при этом $p$ - раздутие прямой в слое, $X^{\prime}$ (локально) гладко и $p^{\prime}$ - стягивание собственного прообраза соответствующего слоя в точку.

5) $\rho(X)=\rho\left(X^{\prime}\right) \geqslant 2, \operatorname{dim}(S)=2, \operatorname{dim}\left(S^{\prime}\right)=2, p, p^{\prime}-$ pаздутия кривых $C$ и $C^{\prime}$, изоморфно проечирующихся на свои образы $\phi(C)$ и $\phi^{\prime}\left(C^{\prime}\right), \chi-$ изоморфизм. При этом исключительным дивизором $p^{\prime}$ является собственный прообраз при р дивизора $D=\phi^{*}(\phi(C)), X$ гладко в точках $D$, а $S$ гладко в точках $\phi(C)$.

СХемА докАЗАТЕЛЬСТВА. Пусть $S=p t, \mathbb{F}_{X}^{(1)}=\left\{v_{1}, \ldots, v_{4}\right\}, \mathbb{F}_{Z}^{(1)}=\mathbb{F}_{Z^{\prime}}^{(1)}=\mathbb{F}_{X}^{(1)} \cup$ $\left\{v_{5}\right\}, \mathbb{F}_{X^{\prime}}^{(1)}=\mathbb{F}_{Z^{\prime}}^{(1)} \backslash\left\{v_{4}\right\}$. Так как многообразие $X^{\prime}$ снова должно быть одним из восьми перечисленных в конще раздела 3 , то для $v_{5}$ при фиксированном $X$ есть лишь конечное число возможностей. Как и в доказательстве предложения 1 , окончательньй ответ в этом случае получается конечным перебором.

В случае $\operatorname{dim}(S)=1$ локальное описание многообразия $X$ дается леммой 3 . Если $X$ особо, $p$ описьвается теоремой Каваматы; если $X$ неособо, ограничения на $p$ возникают из требования терминальности особенностей $Z$.

Если $\operatorname{dim}(S)=2$, то образ $p$ не может содержаться в слое $\phi$, что легко увидеть, например, из строения $\mathbb{F}_{X}$. Оставшаяся часть описания получается применением теоремы Каваматы.

Наконец, самая малочисленная и наиболее просто описываемая группа - линки типа IV. Отметим, что среди них встречаются линки с $\chi=\mathrm{id}$, которые являются просто выбором другой структуры расслоения Мори на многообразии.

ПРЕДЛОЖЕНИЕ 3. Пусть $X \rightarrow S, X^{\prime} \rightarrow S^{\prime}-$ трехмерные торические Мори расслоения. Тогда для линков типа IV имеются лищь следующие возможсности.

1) $\rho(X)=2, \operatorname{dim}(S)=\operatorname{dim}\left(S^{\prime}\right)=1, T=p t, \chi-$ один лог-флип. Cуществует лишь один такой линк, причем $X$ и $X^{\prime}$ изоморфны $\mathbb{P}_{\mathbb{P}^{1}}(\mathscr{O} \oplus \mathscr{O}(1) \oplus \mathscr{O}(1))$.

2) $\rho(X)=2, \operatorname{dim}(S)=1, \operatorname{dim}\left(S^{\prime}\right)=2$ (или наоборот), $T=p t, \chi-$ изоморфизм. Существует лишь два таких линка. В первом из них $X=\mathbb{P}^{2} \times \mathbb{P}^{1}$, а во втором $X=\mathbb{P}^{2} \times \mathbb{P}^{1} / \mathbb{Z}_{3}(2: 1: 0,1: 0)$.

3) $\rho(X)=3, \operatorname{dim}(S)=\operatorname{dim}\left(S^{\prime}\right)=2, \operatorname{dim}(T)=1, \chi-$ изоморфизм. Таких линков бесконечно много, но с локальной точки зрения возможсны лишь две различных ситуации: локально (по T) $X$ изоморфно $\mathbb{P}^{1} \times \mathbb{P}^{1} \times \mathbb{A}^{1}$ или $\mathbb{P}^{1} \times \mathbb{P}^{1} \times \mathbb{A}^{1} / \mathbb{Z}_{2}(1: 0,1: 0,1) c$ естественными проекииями на два әкземпляра $\mathbb{P}^{1} \times \mathbb{A}^{1}\left(\right.$ соответственно $\mathbb{P}^{1} \times \mathbb{A}^{1} /$ $\left.\mathbb{Z}_{2}(1: 0,1)\right)$ и дальнейшими проекииями на $\mathbb{A}^{1}$. 
СХЕМА ДОКАЗАТЕЛЬСТВА. Пусть $T=p t$. Из строения $\mathbb{F}_{X}$ очевидно, что случай $\operatorname{dim}(S)=\operatorname{dim}\left(S^{\prime}\right)=2$ не реализуется. Если $\operatorname{dim}(S)=1, \operatorname{dim}\left(S^{\prime}\right)=2$, то комбинаторная структура $\mathbb{F}_{X}$ однозначно определена тем, что $\mathbb{F}_{X}^{(1)}$ содержит пару коллинеарных и тройку компланарных векторов. Применение леммы 3 заканчивает описание в этом случае.

Предположим, что $\operatorname{dim}(S)=\operatorname{dim}\left(S^{\prime}\right)=1$. Пусть $\mathbb{F}_{X}^{(1)}=\left\{v_{1}, \ldots, v_{5}\right\}$. Так как $\mathbb{F}_{X^{\prime}}^{(1)}=\mathbb{F}_{X}^{(1)}$, а $\mathbb{F}_{X}$ и $\mathbb{F}_{X^{\prime}}$ обладают проекциями на $\mathbb{F}_{\mathbb{P}^{1}}$, то $\mathbb{F}_{X}^{(1)}$ содержит две тройки компланарных векторов, например, $v_{1}, v_{2}, v_{3}$ и $v_{3}, v_{4}, v_{5}$. Так как особенности $X$ и $X^{\prime}$ терминальны, то $v_{1}+v_{2}+v_{3}=0$ и $v_{3}+v_{4}+v_{5}=0$. Можно считать, что $v_{1}=(1,0,0)$, $v_{2}=(0,1,0), v_{3}=(-1,-1,0)$. По лемме 3 можно считать, что либо $v_{4}=(0,0,1)$ (тогда $v_{5}=(1,1,-1)$ и $\left.X=\mathbb{P}_{\mathbb{P}^{1}}(\mathscr{O} \oplus \mathscr{O}(1) \oplus \mathscr{O}(1))\right)$, либо $v_{4}=(1,2,3)$ (тогда $v_{5}=(0,-1,-3)$ и $X$ имеет не терминальные особенности; противоречие).

Если $\operatorname{dim}(T)=1$, то локальное описание $X$ и $X^{\prime}$ получается из леммы 4 . Случай $\operatorname{dim}(T)=2$, как нетрудно видеть, не реализуется.

ЗАмЕчаниЕ 4. Отметим, что во всех перечисленных случаях отображение $\chi$ либо тривиально, либо состоит из одного лог-флипа.

ЗАмЕЧАнИЕ 5. Хотя самьй простой способ доказательства приведенных вьше утверждений комбинаторный, некоторые из них верны и в общем (не торическом) случае. Таковы, например, утверждения о том, что линков типа IV c $\operatorname{dim}(T)=2$, а также линков типа I c $\operatorname{dim}(S)=1$, для которых $p$ - раздутие подмногообразия слоя $\phi$, в случае трехмерных Мори расслоений не существует.

ЗАмЕЧАнИЕ 6. Некоторые из приведенных выше линков можно описать более явно, как, например, линк из пункта 1) предложения 3. Пусть $M$ - тавтологический обратимый пучок на $X=\mathbb{P}_{\mathbb{P}^{1}}(\mathscr{O} \oplus \mathscr{O}(1) \oplus \mathscr{O}(1))$, дивизор $L$ - слой расслоения $\phi: X \rightarrow \mathbb{P}^{1}$. Пусть $l$-прямая в слое, $s$ - сечение, соответствующее проекции расслоения $\mathscr{O} \oplus \mathscr{O}(1) \oplus \mathscr{O}(1)$ на $\mathscr{O}$. Тогда $L s=1, L l=0, M s=0, M l>0$, т.е. $s$ и $l$ порождают экстремальные лучи конуса Мори $N E(X)$. Так как $K_{X}=-3 M$, то $K_{X} s=0$. В кривой $s$ можно сделать флоп. Именно он и является отображением $\chi$. Подробное описание этого флопа можно найти, например, в [5] (там он описьвается как переход от одного малого разрешения особенности конуса над квадрикой $\mathbb{P}^{1} \times \mathbb{P}^{1}$ к другому малому разрешению).

Автор выражает благодарность В.А. Исковских и Ю.Г. Прохорову за постановку задачи и внимание к работе.

\section{СПИСОК ЦИТИРОВАННОЙ ЛИТЕРАТУРЫ}

[1] Matsuki K. Introduction to the Mori Program. Universitext. New York: Springer-Verlag, 2002.

[2] Данилов В. И. Геометрия торических многообразий // УМН. 1978. Т. 33. № 2. С. 85-134.

[3] Kawamata Y. Divisorial contractions to 3-dimensional terminal quotient singularities // Higher-Dimensional Complex Varieties (Trento, 1994). Berlin: de Gruyter, 1996. P. 241-246.

[4] Борисов А. А., Борисов Л. А. Особые торические многообразия Фано // Матем. сб. 1992. T. 183. № 2. C. 134-141.

[5] Клеменс Х., Коллар Я., Мори С. Многомерная комплексная геометрия. М.: Мир, 1993.

Московский государственный университет им. М.В. Ломоносова

E-mail : shramov@mccme.ru

Поступило

09.03.2004 